УДК 519.6

М. Ю. Ракушев, к.т.н., с.н.с.

\title{
ВАРІАНТ НЕЯВНОЇ СХЕМИ ЧИСЛОВОГО ІНТЕГРУВАННЯ НА ОСНОВІ ЗМЩЕНИХ ДИФЕРЕНЦІАЛЬНИХ ПЕРЕТВОРЕНЬ
}

\author{
Національний авіаційний університет \\ E-mail: r16mu0977@meta.ua
}

Запропоновано неявну обчислювальну схему розв'язання задачі Коші для звичайного диференціального рівняння на основі зміщених диференціальних перетворень із використанням ступеневих функиій. Показано, щуо схема має оптимальний порядок точності і є А-стійкою, що дозволяє ефективно застосовувати ї̈ для розв'язання жорстких диференціальних рівнянь.

Ключові слова: диференціальні перетворення, задача Коші, числові схеми.

\section{Постановка проблеми}

У багатьох практичних задачах, наприклад, з областей хімічної кінетики, процесів управління та теорії електричних кіл, виникає необхідність розв’язання задачі Коші для жорсткого диференціального рівняння.

Розв'язання такої задачі проводиться одним із методів числового інтегрування звичайних диференціальних рівнянь шляхом розробки відповідної неявної обчислювальної схеми інтегрування $[1 ; 2 ; 3]$.

Можливим варіантом розв'язання жорсткої задачі Коші є використання операційного методу диференціальних перетворень [4].

\section{Аналіз останніх досліджень}

Питання розробки неявних обчислювальних схем на основі диференціальнотейлорівських перетворень (далі Т-схеми) розглянуто в роботах $[4 ; 5 ; 6 ; 7 ; 8]$.

$\mathrm{У}$ роботах $[7 ; 8]$ показано, що введення апроксимаційних поправок (для схеми, наведеної в роботі [6]) або проведення взаємного врахування основного i зміщеного Т-спектрів (для схем, наведених в роботах [4;8]) приводить до збільшення точності та значного розширення області стійкості таких схем. При цьому найкращі характеристики зі стійкості - А-стійкість - у неявної (зміщеної) Т-схеми [8], а з точності - у неявної (апроксимаційної) Т-схеми [6].
Порядок точності неявної (зміщеної) Т-схеми дорівнює номеру старшої враховуваної в ній Т-дискрети, якщо вона парна, або на одиницю більший номера старшої враховуваної в ній Т-дискрети, якщо вона непарна [8].

Порядок точності такої схеми у два рази менший, чим загальна кількість ураховуваних у ній (в основному та зміщеному Т-спектрах) Т-дискрет.

Можливим шляхом підвищення порядку точності при збереженні характеристик зі стійкості неявної (зміщеної) Т-схеми $є$ введення в таку схему додаткових (апроксимаційних) умов.

Мета роботи - розробка А-стійкої з оптимальним (не покращувальним) порядком точності неявної обчислювальної схеми розв'язання задачі Коші для звичайного диференціального рівняння на основі зміщених диференціальних перетворень.

\section{Виклад основного матеріалу}

Задача Коші для звичайного диферентіального рівняння має вигляд:

$$
\frac{d u}{d t}=f(t, u), \quad t>0, \quad u(t=0)=u_{0},
$$

де $f(t, u)$ - задана функція, безперервно диференційована за $t$ та $u$;

$$
\begin{aligned}
& t \text { - незалежна змінна; } \\
& u \text { - шукана функція; }
\end{aligned}
$$


$u_{0}$ - початкова умова.

Диференціальними перетвореннями називають функціональні перетворення вигляду [4]:

$$
\begin{aligned}
& Z(k)=\frac{H^{k}}{k !}\left[\frac{d^{k} z(t)}{d t^{k}}\right]_{t_{*}}=\frac{H^{k}}{k !} z^{(k)}\left(t_{*}\right), \\
& z(t)=\varphi(t),
\end{aligned}
$$

де $Z(k)$ - дискретна функція за аргументом $k$;

$H$ - відрізок аргументу, на якому розглядається функція $z(t)$;

$k$ - цілочисловий аргумент $k=0,1$;

$t$ - аргумент, за яким проводиться перетворення;

$t_{*}$ - значення аргументу, при якому проводиться перетворення;

$\varphi(t)$ - відновлююча функція.

Вирази (2) визначають пряме і обернене диференціальні перетворення. Множину значень $Z(k)$ прийнято називати Т-спектром, а значення функції $Z(k)$ при конкретних значеннях аргументу $k$-дискретами Т-спектра.

У найпростішому випадку для відновлення використовують відрізок ряду Тейлора i такі перетворення називають диференціально-тейлорівськими [4]:

$$
z(t) \approx \sum_{k=0}^{k_{\max }}\left(\frac{t-t_{*}}{H}\right)^{k} Z(k),
$$

де $k_{\max }$ - максимальний номер ураховуваної при відновленні Т-дискрети.

Неявна (зміщена) Т-схема числового розв'язання задачі (1) на основі диферентіально-тейлорівьких перетворень (3) - це обчислювальна схема, в якій значення функції у точці $n+1$ розраховується так, щоб $k_{\max }$-та часткова сума ряду Тейлора, обчисленого в ній, - сума дискрет зміщеного Т-спектра, дорівнювала на середині інтервалу $\left[t_{n}, t_{n+1}\right]$ $k_{\max }$-частковій сумі дискрет ряду Тейлора, обчисленого в попередній точці $n$ - сумі дискрет основного Т-спектра [8]:

$$
\begin{aligned}
& \left\{\begin{array}{l}
Y_{0}(k=0)=u_{0}, \quad H_{n}=t_{n+1}-t_{n} ; \\
Y_{n}(k+1)=\frac{H_{n}}{k+1} F_{n}(k) ; \\
Y_{n+1}(k+1)=\frac{H_{n}}{k+1} F_{n+1}(k) ;
\end{array}\right. \\
& \sum_{k=0}^{k_{\max }}\left(-\frac{1}{2}\right)^{k} Y_{n+1}(k)=\sum_{k=0}^{k_{\max }}\left(\frac{1}{2}\right)^{k} Y_{n}(k),
\end{aligned}
$$

де $Y_{n}(k), Y_{n+1}(k), F_{n}(k), F_{n+1}(k)$ - Т-спектри розв'язку задачі (1) та правої частини диференціального рівняння (1) у відповідних точках обчислювальної сітки

$$
\omega_{n}=\left\{t_{n}, n=0,1,2, \ldots\right\}
$$

(точка $n$ - основний Т-спектр, точка $n+1-$ зміщений Т-спектр).

Залежності (4), (5), дозволяють послідовно (починаючи $3 n=0$ ) знайти розв'язок рівняння (1) - визначити на $\omega_{n}$ значення сіткової функції

$$
y_{n}=Y_{n}(0),
$$

яке приймається за наближення шуканої функції

$$
u\left(t_{n}\right) \approx y_{n} .
$$

Додатково, спираючись на властивості Т-спектра $Y_{n}(k)$, визначається наближення $u(t)$ у вигляді кусково-безперервної аналітичної функції між вузлами сітки $\omega_{n}$ [4].

Уведемо в операцію відновлення (5) Т-схеми (4), (5) апроксимаційні коефіцієнти, 3 урахуванням яких отримаємо неявну схему числового інтегрування на основі зміщених нетейлорівських перетворень [4] із використанням ступеневих функції

$$
\sum_{k=0}^{m} a_{k} Y_{n+1}(k)=\sum_{k=0}^{r} b_{k} Y_{n}(k) \text {. }
$$

де $a_{k}, b_{k}$ - апроксимаційні коефіцієнти;

$m, r$ - максимальні номери дискрети, що враховуються при відновленні в основному і зміщеному Т-спектрах відповідно.

Розпишемо рівняння (5) 3 урахуванням прямого диференціального перетворення 3 виразу (2):

$$
\sum_{k=0}^{m} a_{k} \frac{H_{n}^{k}}{k !} y_{n+1}^{(k)}=\sum_{k=0}^{r} b_{k} \frac{H_{n}^{k}}{k !} y_{n}^{(k)} .
$$


Приведемо рівність (5) до канонічного вигляду обчислювальної схеми числового інтегрування прийнятої для скінченнорізницевих методів [1].

Додатково, для спрощення подальших викладок введемо заміну $h=H_{n}$ :

$$
\begin{aligned}
& \frac{a_{0} y_{n+1}-b_{0} y_{n}}{h}= \\
& =\frac{1}{h}\left(\sum_{k=1}^{r} b_{k} \frac{h^{k}}{k !} y_{n}^{(k)}-\sum_{k=1}^{m} a_{k} \frac{h^{k}}{k !} y_{n+1}^{(k)}\right) .
\end{aligned}
$$

Визначимо похибку апроксимації $\psi_{n}$ отриманої схеми (4), (7). Для цього підставимо у рівнянні (7) замість числового (наближеного) розв'язку $-y_{n}$ точний розв'язок задачі (1) $-u=u(t)$ у вигляді розкладу в точці $t=t_{n}$ за формулою Тейлора [1]:

$$
\begin{aligned}
& u_{n+1}=\sum_{s=0}^{p} \frac{h^{s}}{s !} u_{n}^{(s)}+O\left(h^{p+1}\right), \\
& u_{n+1}^{(k)}=\sum_{d=k}^{p} \frac{h^{d-k}}{(d-k) !} u_{n}^{(d)}+O\left(h^{p-k+1}\right) \Rightarrow \\
& \Rightarrow \psi_{n}=\frac{1}{h}\left(\sum_{k=0}^{r} b_{k} \frac{h^{k}}{k !} u_{n}^{(k)}-\sum_{k=0}^{m} a_{k} \frac{h^{k}}{k !} u_{n+1}^{(k)}\right) \Rightarrow \\
& \Rightarrow \psi_{n}=\frac{1}{h}\left(\sum_{k=0}^{r} b_{k} \frac{h^{k}}{k !} u_{n}^{(k)}-\right. \\
& \left.-\sum_{k=0}^{m}\left[\frac{a_{k}}{k !}\left(\sum_{d=k}^{p} \frac{h^{d}}{(d-k) !} u_{n}^{(d)}\right)\right]\right)+O\left(h^{p}\right) .
\end{aligned}
$$

Покладемо $p>\max (m, r) .3$ урахуванням цього формулу (8) можна перетворити до вигляду

$$
\begin{aligned}
& \psi_{n}=\frac{1}{h}\left(\sum_{k=0}^{r} b_{k} \frac{h^{k}}{k !} u_{n}^{(k)}-\right. \\
& \left.-\sum_{d=0}^{p} h^{d} u_{n}^{(d)}\left[\sum_{k=0}^{\min (d, m)} \frac{a_{k}}{k !} \frac{1}{(d-k) !}\right]\right)+O\left(h^{p}\right) .
\end{aligned}
$$

Змінимо індекс у першій сумі з $k$ на $d$ :

$$
\begin{aligned}
\psi_{n} & =\frac{1}{h}\left(\sum_{d=0}^{r} b_{d} \frac{h^{d}}{d !} u_{n}^{(d)}-\right. \\
& \left.-\sum_{d=0}^{p} h^{d} u_{n}^{(d)}\left[\sum_{k=0}^{\min (d, m)} \frac{a_{k}}{k !} \frac{1}{(d-k) !}\right]\right)+O\left(h^{p}\right) \Rightarrow
\end{aligned}
$$

$\Rightarrow \psi_{n}=\frac{1}{h}\left(\sum_{d=0}^{r} h^{d} u_{n}^{(d)}\left[\frac{b_{d}}{d !}-\sum_{k=0}^{\min (d, m)} \frac{a_{k}}{k !} \frac{1}{(d-k) !}\right]-\right.$

$\left.-\sum_{d=r+1}^{p} h^{d} u_{n}^{(d)} \sum_{k=0}^{\min (d, m)} \frac{a_{k}}{k !} \frac{1}{(d-k) !}\right)+O\left(h^{p}\right)$.

Із формули (9) видно, що похибка апроксимації схеми, що розглядається, має порядок $p$, якщо виконані умови

$\left\{\begin{array}{l}\sum_{k=0}^{\min (d, m)} a_{k} \frac{d !}{k !(d-k) !}=b_{d}, \text { якщо } d=0, \cdots, r \\ \sum_{k=0}^{\min (d, m)} a_{k} \frac{d !}{k !(d-k) !}=0, \text { якщо } d=r+1, \cdots, p\end{array}\right.$

У системі (10) при $d=0$ отримаємо $a_{0}=b_{0}$, тобто (10) має нескінченну кількість розв'язків.

Для усунення такої невизначеності достатньо покласти $a_{0}=1$. Для того, щоб система (10) не була перевизначена, необхідно накласти вимогу

$$
p=m+r \text {. }
$$

Введемо в систему (11) заміну

$$
a_{k}^{*}=\frac{a_{k}}{k !}, b_{d}^{*}=\frac{b_{d}}{d !},
$$

з врахуванням якої запишемо

$$
\left\{\begin{array}{l}
\sum_{k=0}^{\min (d, m)} a_{k}^{*} \frac{1}{(d-k) !}=b_{d}^{*} \text {, якщо } d=0, \cdots, r \\
\sum_{k=0}^{\min (d, m)} a_{k}^{*} \frac{1}{(d-k) !}=0, \text { якщо } d=r+1, \cdots, r+m
\end{array}\right.
$$

Система (13) визначає коефіцієнти апроксимації Паде для функції [9]

$$
\exp (x)=\frac{\sum_{d=0}^{r} b_{d}^{*} x^{d}}{1+\sum_{k=1}^{m} a_{k}^{*} x^{k}}+O\left(x^{r+m+1}\right),
$$

в якій

$a_{k}^{*}=\frac{(-1)^{k}}{k !} \frac{(r+m-k) !}{(r+m) !} \frac{m !}{(m-k) !}$, якщо $k=1, \cdots, m$,

$b_{d}^{*}=\frac{1}{d !} \frac{(r+m-d) !}{(r+m) !} \frac{r !}{(r-d) !}$, якщо $d=0, \cdots, r$.

3 урахуванням рівностей (12), розв'язок системи (10) матиме вигляд 


$$
\begin{gathered}
a_{k}=(-1)^{k} \frac{(r+m-k) !}{(r+m) !} \frac{m !}{(m-k) !}, \text { якщо } k=0, \cdots, m, \\
b_{d}=\frac{(r+m-d) !}{(r+m) !} \frac{r !}{(r-d) !}, \text { якщо } d=0, \cdots, r .
\end{gathered}
$$

Отже, якщо коефіцієнти обчислювальної схеми (4), (6) розраховані відповідно до системи (16), то порядок іiі апроксимації буде дорівнювати сумі максимальних номерів ураховуваних у ній Т-дискрет основного та зміщеного Т-спектрів (11), з головним членом асимптотичного розкладу похибки апроксимації [2; 3], відповідно до виразів (9), (10):

$$
\psi_{n}=O\left(h^{m+r}\right) \approx E_{m, r} \frac{h^{m+r}}{(m+r+1) !} u_{n}^{(m+r+1)},
$$

де $E_{m, r}$ - коефіцієнт, що характеризує похибку обчислювальної схеми:

$$
E_{m, r}=-\sum_{k=0}^{m}(-1)^{k} \frac{(m+r+1)}{(m+r+1-k)} \frac{m !}{(m-k) ! k !} .
$$

Дослідимо стійкість отриманої схеми (4), (6), (16). Для цього розглянемо модельне рівняння $[1 ; 2]$ :

$$
\frac{d u}{d t}=\lambda u,
$$

де $\lambda$ - довільне комплексне число.

Застосування формул (4), (6), (16) до рівняння (18) дасть різницеве рівняння досліджуваної обчислювальної схеми у вигляді

$$
\begin{aligned}
Y_{n+1}(0) & =\frac{\sum_{k=0}^{r} b_{k} \frac{(\lambda h)^{k}}{k !}}{\sum_{k=0}^{m} a_{k} \frac{(\lambda h)^{k}}{k !}} Y_{n}(0) \Rightarrow \\
\Rightarrow y_{n+1} & =R(\mu) y_{n}, R(\mu)=\frac{\sum_{k=0}^{r} b_{k} \frac{\mu^{k}}{k !}}{\sum_{k=0}^{m} a_{k} \frac{\mu^{k}}{k !}}
\end{aligned}
$$

де $\mu=\lambda h-$ комплексний параметр;

$R(\mu)$ - функція стійкості отриманої обчислювальної схеми [2].

Відповідно до формул (14), (15), (16) функція стійкості розробленої схеми $R(\mu) \epsilon$ апроксимацією Паде експоненціальної функції. Методи, $R(\mu)$ яких є елементом діагоналі, або однією 3 двох головних піддіагоналей таблиці Паде експоненціальної функції, є А-стійкими [2].
Отже, всі обчислювальні схеми вигляду (4), (6), (16) для $m=r, m=r+1, m=r+2 \epsilon$ А-стійкими.

При цьому для схеми (19), якщо $m=r+1$ або $m=r+2$, виконується умова L-стійкості: $\lim _{|\mu| \rightarrow \infty}|R(\mu)|=0$, якщо $m=r+1$ або $m=r+2$,

яка є найбільш бажаною для жорстких задач [2] (порівняно для $m=r$ і для Т-схеми (4), (5)

$$
\left.\lim _{|\mu| \rightarrow \infty}|R(\mu)|=1\right) .
$$

Запропонована схема числового інтегрування є однокроковою, стійкою та апроксимує вихідне диференціальне рівняння, і тому вона збігається, якщо $h \rightarrow 0$, до точного розв'язку диференціального рівняння, причому порядок точності схеми збігається 3 ïi порядком апроксимації (11) [2].

Використовуючи функцію стійкості $R(\mu)$, можна побудувати області стійкості для розробленої обчислювальної схеми [2].

Зазначені області стійкості, для різних $m$ i $r$ показано на рисунку, де числа - 1-15 позначають порядок точності відповідної схеми, який дорівнює $m+r$.

Для більшої наочності області стійкості (зовнішні області) зафарбовано у темні кольори.

Значення коефіцієнтів (16), (17) для різних варіантів запропонованої обчислювальної схеми наведено у табл. 1-3.

Запропонована схема (4), (6), (16) є неявною. Це означає, що для нелінійного диференціального рівняння (1) вона на кожному кроці обчислювальної сітки $\omega_{n}$ зводиться до нелінійного рівняння відносно $y_{n+1}$, розв'язати яке можна, наприклад, методом Ньютона:

$$
\begin{aligned}
& \left(y_{n+1(i+1)}-y_{n+1(i)}\right) \sum_{k=0}^{m_{1}} a_{k} \delta Y_{n+1(i)}(k)= \\
& =\sum_{k=0}^{r} b_{k} Y_{n}(k)-\sum_{k=0}^{m} a_{k} Y_{n+1(i)}(k),
\end{aligned}
$$

де $i$ - номер ітерації за методом Ньютона;

$m_{1}$ - максимальний номер Т-дискрети, що враховується в Т-спектрі матриці Якобі;

$\delta Y_{n+1(i)}(k)=\frac{\partial Y_{n+1(i)}(k)}{\partial y_{n+1(i)}}-$ Т-спектр матриці

Якобі. 


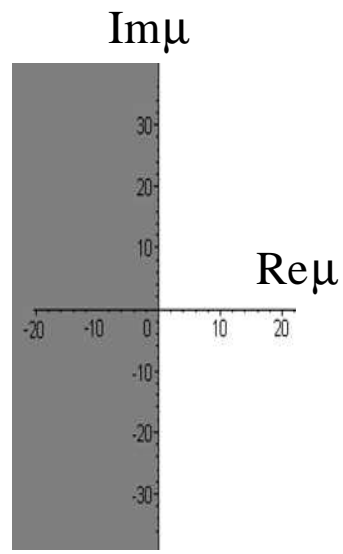

$a$

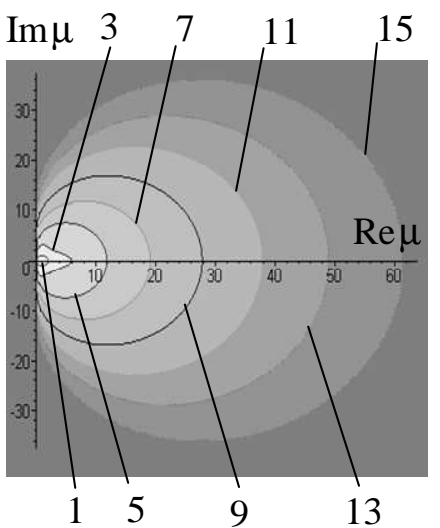

6

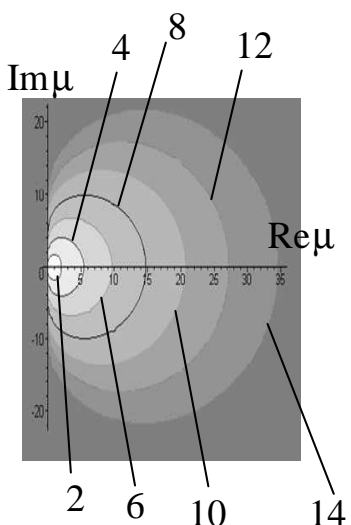

B

Рис. 1. Області стійкості запропонованої обчислювальної схеми:

$a-$ для $m=r$;

$\sigma$ - для $m=r+1$;

в - для $m=r+2$

Таблиця 1

Табличя 2

Коефіцієнти розробленої схеми для $m=r$

\begin{tabular}{|c|c|c|c|c|c|c|c|c|}
\hline \multirow{2}{*}{$\begin{array}{c}\text { Поря- } \\
\text { док } \\
\text { точно- } \\
\text { сті }\end{array}$} & \multirow{2}{*}{$\begin{array}{l}\text { Koе- } \\
\text { фіці- } \\
\epsilon \text { нт }\end{array}$} & \multicolumn{6}{|c|}{$k$} & \multirow{2}{*}{$E_{m, 1}$} \\
\hline & & 0 & 1 & 2 & 3 & 4 & 5 & \\
\hline \multirow{2}{*}{2} & $a_{k}$ & 1 & $\frac{-1}{2}$ & & - & - & - & \multirow{2}{*}{$\frac{1}{2}$} \\
\hline & $b_{k}$ & 1 & $\frac{1}{2}$ & & - & - & - & \\
\hline \multirow{2}{*}{4} & $a_{k}$ & 1 & -1 & $\frac{1}{6}$ & $=$ & - & - & \multirow{2}{*}{$\frac{-1}{6}$} \\
\hline & $b_{k}$ & 1 & 1 & $\frac{1}{6}$ & - & - & - & \\
\hline \multirow{2}{*}{6} & $a_{k}$ & 1 & $\frac{-1}{2}$ & $\frac{1}{5}$ & $\frac{-1}{20}$ & - & - & \multirow{2}{*}{$\underline{1}$} \\
\hline & $b_{k}$ & 1 & $\frac{1}{2}$ & $\frac{1}{5}$ & $\frac{1}{20}$ & - & - & \\
\hline \multirow{2}{*}{8} & $a_{k}$ & 1 & $\frac{-1}{2}$ & $\frac{3}{14}$ & $\frac{-1}{14}$ & $\frac{1}{70}$ & - & \multirow{2}{*}{$\frac{-1}{70}$} \\
\hline & $b_{k}$ & 1 & $\frac{1}{2}$ & $\frac{3}{14}$ & $\frac{1}{14}$ & $\frac{1}{70}$ & - & \\
\hline \multirow[t]{2}{*}{10} & $a_{k}$ & 1 & $\frac{-1}{2}$ & $\frac{2}{9}$ & $\frac{-1}{12}$ & $\frac{1}{42}$ & $\frac{-1}{252}$ & \multirow{2}{*}{$2 \frac{1}{52}$} \\
\hline & $b_{k}$ & 1 & $\frac{1}{2}$ & $\frac{2}{9}$ & $\frac{1}{12}$ & $\frac{1}{42}$ & $\frac{1}{252}$ & \\
\hline
\end{tabular}

Коефіцієнти розробленої схеми для $m=r+1$

\begin{tabular}{|c|c|c|c|c|c|c|c|c|c|}
\hline \multirow{2}{*}{$\begin{array}{c}\text { Поря- } \\
\text { док } \\
\text { точно- } \\
\text { сті }\end{array}$} & \multirow{2}{*}{$\begin{array}{l}\text { Кое- } \\
\text { фіці- } \\
\text { єнт }\end{array}$} & \multicolumn{7}{|c|}{$k$} & \multirow{2}{*}{$E_{m, n}$} \\
\hline & & 0 & 1 & 2 & 3 & 4 & 5 & 6 & \\
\hline \multirow{2}{*}{3} & $a_{k}$ & 1 & $\frac{2}{3}$ & $\frac{1}{3}$ & - & - & - & - & \multirow{2}{*}{$\frac{5}{3}$} \\
\hline & $b_{k}$ & 1 & $\frac{1}{3}$ & - & - & - & - & - & \\
\hline \multirow{2}{*}{5} & $a_{k}$ & 1 & $\frac{3}{5}$ & $\frac{3}{10}$ & $\frac{1}{10}$ & - & - & - & \multirow{2}{*}{$\frac{-19}{10}$} \\
\hline & $b_{k}$ & 1 & $\frac{2}{5}$ & $\frac{1}{10}$ & - & - & - & - & \\
\hline \multirow{2}{*}{7} & $a_{k}$ & 1 & $\frac{4}{7}$ & $\frac{2}{7}$ & $\underline{4}$ & $\frac{1}{35}$ & - & - & \multirow{2}{*}{$\frac{69}{35}$} \\
\hline & $b_{k}$ & 1 & $\frac{3}{7}$ & $\frac{1}{7}$ & $\frac{1}{35}$ & - & - & - & \\
\hline \multirow{2}{*}{9} & $a_{k}$ & 1 & $\frac{5}{9}$ & $\underline{5}$ & $\frac{5}{42}$ & $\underline{5}$ & $\frac{1}{126}$ & - & -251 \\
\hline & $b_{k}$ & 1 & $\frac{4}{9}$ & $\frac{1}{6}$ & $\frac{1}{21}$ & $\frac{1}{126}$ & - & - & 126 \\
\hline \multirow{2}{*}{11} & $a_{k}$ & 1 & $\underline{6}$ & $\frac{3}{11}$ & $\frac{4}{33}$ & $\frac{1}{22}$ & $\frac{1}{77}$ & $\frac{1}{462}$ & \multirow{2}{*}{$\frac{923}{462}$} \\
\hline & $b_{k}$ & 1 & $\frac{5}{11}$ & $\frac{2}{11}$ & $\frac{2}{33}$ & $\frac{1}{66}$ & $\frac{1}{462}$ & - & \\
\hline
\end{tabular}


Таблиця 3

Коефіціснти розробленої схеми для $m=r+2$

\begin{tabular}{|c|c|c|c|c|c|c|c|c|}
\hline \multirow{2}{*}{\begin{tabular}{|c|} 
Поря- \\
док точ \\
ності
\end{tabular}} & \multirow{2}{*}{$\begin{array}{l}\text { Коефі- } \\
\text { цієнт }\end{array}$} & \multicolumn{6}{|c|}{$k$} & \multirow{2}{*}{$E_{m, r}$} \\
\hline & & 0 & 1 & 2 & 3 & 4 & 5 & \\
\hline \multirow{2}{*}{2} & $a_{k}$ & 1 & 1 & 1 & - & - & - & \multirow{2}{*}{-1} \\
\hline & $b_{k}$ & 1 & - & - & - & - & - & \\
\hline \multirow{2}{*}{4} & $a_{k}$ & 1 & $\frac{3}{4}$ & $\frac{1}{2}$ & $\frac{1}{4}$ & - & - & \multirow{2}{*}{$\frac{11}{4}$} \\
\hline & $b_{k}$ & 1 & $\frac{1}{4}$ & - & - & - & - & \\
\hline \multirow{2}{*}{6} & $a_{k}$ & 1 & $\frac{2}{3}$ & $\frac{2}{5}$ & $\frac{1}{5}$ & $\frac{1}{15}$ & - & \multirow{2}{*}{$\frac{-71}{15}$} \\
\hline & $b_{k}$ & 1 & $\frac{1}{3}$ & $\frac{1}{15}$ & - & - & - & \\
\hline \multirow{2}{*}{8} & $a_{k}$ & 1 & $\frac{5}{8}$ & $\frac{5}{14}$ & $\frac{5}{28}$ & $\frac{1}{14}$ & $\frac{1}{56}$ & \multirow{2}{*}{$\frac{379}{56}$} \\
\hline & $b_{k}$ & 1 & $\frac{3}{8}$ & $\frac{3}{28}$ & $\frac{1}{56}$ & - & - & \\
\hline
\end{tabular}

Початкове наближення для $i=0$ при реалізації схеми (20) можна отримати, використовуючи традиційну (явну) Т-схему [4].

Т-спектр матриці Якобі визначають, використовуючи підхід, викладений у роботі [10], або метод скінченних різниць.

Виходячи зі збіжності ітераційного процесу, для зменшення результуючої обчислювальної складності в формулі (20) допускається задавати $m_{1}<m$. Наприклад, якщо $m_{1}=0$, отримаємо метод простої ітерації [1], оскільки $\delta Y_{n+1(i)}(0)=I-$ одинична матриця [10].

\section{Висновки}

Розроблено схему числового інтегрування (4), (6), (16) універсального вигляду, оскільки вона включає багато з відомих Т-схем.

Так, якщо $m=0, b_{k}=1$, маємо (найпростішу) явну Т-схему [4], якщо $r=0, a_{k}=1$, неявну Т-схему [5], якщо $r=m$, $(-1)^{k} a_{k}=b_{k}=2^{-k}, \quad-$ неявну (зміщену) Т-схему (4), (5).
Порядок точності розробленої схеми (3), (14), (19) дорівнює сумі максимальних номерів Т-дискрет основного i зміщеного Т-спектрів, що беруть участь в операції відновлення.

Кожен урахований член ряду Тейлора (кожна Т-дискрета) точного розв'язку диференціального рівняння підвищує порядок точності схеми на одиницю.

Отже, розроблена схема має не покращуваний (оптимальний) для схем вигляду (4), (6) порядок точності. При цьому вид похибки апроксимації розробленої обчислювальної схеми (17) подібний до виду такої похибки для лінійних багатокрокових методів, а ii значення для розробленої схеми (значення коефіцієнта $E_{m, r}$ ) менше такого значення для лінійних багатокрокових методів [3].

Розроблена схема для $m=r \in \mathrm{A}$-стійкою, а для $m=r+1, m=r+2-\mathrm{L}$-стійкою.

Вигляд іiі області абсолютної стійкості повністю збігається 3 виглядом такої області для методів Рунге-Кутта оптимального порядку [2], оскільки розроблена схема має однакові з ними функції стійкості, які $є$ апроксимаціями Паде для експоненціальної функціï.

Розроблено А-стійку з оптимальним порядком точності неявну обчислювальну схему розв'язання задачі Коші для звичайного диференціального рівняння на основі зміщених диференціальних перетворень 3 використанням степеневих функцій.

Характеристики точності та стійкості запропонованої схеми дозволяють ефективно застосовувати ऑii для розв'язання жорстких диференціальних рівнянь.

\section{Література}

1. Самарский А.А. Численные методы: учеб. пособие для вузов / А.А. Самарский, А.В. Гулин. - М.: Наука. Гл. ред. физ.-мат. лит., 1989. -432 с.

2. Холл Дж. Современные численные методы решения обыкновенных дифференциальных уравнений / Дж. Холл, Дж. Уатт. - М.: Мир, 1979. - 321 с. 
3. Хемминг Р.В. Численные методы для научных работников и инженеров / Р.В. Хемминг. - М.: Наука, 1972. - 400 с.

4. Пухов Г.Е. Дифференциальные преобразования функций и уравнений / Г.Е. Пухов. - К.: Наук. думка, 1980. $420 \mathrm{c}$.

5. Ронто Н.И. О неявных схемах интегрирования, основанных на дифференциальных преобразованиях / Н.И. Ронто // Электронное моделирование. - 1986. - Т. 8, № 4. C. 44-50.

6. Степанов A.B. Аппроксимационный вариант неявной Т-схемы численного интегрирования / А.В. Степанов // Теоретическая электротехника. - 1985. - Вып. 39. C. 123-126.
7. Коваль Н.В. Об устойчивости алгоритмов решения систем обыкновенных дифференциальных уравнений методом дифференциального преобразования / Н.В. Коваль, Э.П. Семагина // Теоретическая электротехника. - 1985. - Вып. 39. - С. 108-118.

8. Ракушев М.Ю. Апроксимація та стійкість методу зміщених диференціально-тейлорівських перетворень для рішення задачі Коші / М.Ю. Ракушев // Вісник ЖДТУ. - Житомир: ЖДТУ, 2007. - 42, № 3. - С. 128-132.

9. Бейкер Дж. мл. Аппроксимации Паде / Дж. Бейкер мл., П. Грейвс-Моррис / пер. с англ. - М.: Мир, 1886.- 502 с.

10. Ковбасюк C.B. Метод решения вариационного уравнения для задачи Коши на основе дифференциальных преобразований / С.В. Ковбасюк, М.Ю. Ракушев // Электронное моделирование. - 2008. - Т. 30, № 6. С. 59-70. 